Check for updates

Cite this: RSC Adv., 2017, 7, 52296

Received 29th September 2017 Accepted 30th October 2017

DOI: $10.1039 / c 7 r a 10786 b$

rsc.li/rsc-advances

\section{A first-principles study of titanium oxide clusters formation and evolution in a steel matrix}

\author{
Wenqiang Bao, (D) ${ }^{a}$ Wei Zhang, ${ }^{b}$ Huigai Li, ${ }^{\star a}$ Shaobo Zheng $^{a}$ and Qijie Zhai ${ }^{a}$ \\ The structures and evolution law of $\mathrm{Ti}_{m} \mathrm{O}_{n}$ clusters in a bcc Fe matrix are studied by using first-principles \\ calculations. In a bcc lattice, the $\mathrm{Ti}$ and $\mathrm{O}$ atoms attract each other strongly when the second nearest \\ neighbor site to interstitial $\mathrm{O}$ has been replaced by $\mathrm{Ti}$. The $\mathrm{Ti}-\mathrm{O}$ and $\mathrm{Ti}-\mathrm{Ti}$ bonds are two dominating \\ factors affecting the stability of clusters. Ti-O bonds have more contributions to the stability of clusters \\ than $\mathrm{Ti}-\mathrm{Ti}$ bonds. The stoichiometric ratio of $\mathrm{Ti}$ to $\mathrm{O}$ tends to stay $1: 1$ in titanium oxide clusters by \\ absorbing $\mathrm{Ti} / \mathrm{O}$ atoms and then the cluster tends to absorb $\mathrm{O}$ atoms. The stable rhombus-shaped \\ structure $\mathrm{Ti}_{2} \mathrm{O}_{2}$ is suggested to be the smallest unit of titanium oxide inclusions in steel. This work \\ provides practical information for further study of oxide inclusion nucleation.
}

\section{Introduction}

Fine titanium oxides such as $\mathrm{TiO}, \mathrm{TiO}_{2}, \mathrm{Ti}_{2} \mathrm{O}_{3}$ and $\mathrm{Ti}_{3} \mathrm{O}_{5}$ can serve as heterogeneous nucleation sites for intragranular ferrite (IGF) in low-alloy steels, which could improve the toughness of steel. ${ }^{1-3}$ It is difficult to study the nucleation mechanism of titanium oxide in experiments due to the high temperature of melt (1400-1500 $\left.{ }^{\circ} \mathrm{C}\right)$. It has been confirmed that first-principles calculation provides a practicable and predictive approach for studying the mechanism of nucleation from the view of atomic crystal structures. Normally, to acquire fundamental insight into titanium oxides nucleation, it is necessary to understand the interaction between $\mathrm{Ti}$ and $\mathrm{O}$ solute elements, the formation of a titanium oxide cluster, and the evolution of the cluster in the bcc Fe matrix.

Previous first-principles calculation studies were focused on the formation and stability of $\mathrm{TiO}_{x}$ clusters without a matrix. Y. Guo et al. found that $\left(\mathrm{TiO}_{2}\right)_{n}$ was the most stable structure among the investigated titanium oxygen species. ${ }^{4}$ Compact structures preferred to form among $\left(\mathrm{TiO}_{2}\right)_{n}$ clusters with increasing cluster size. ${ }^{5}$ D. A. Dixon et al. investigated some equilibrium geometries of defect-free $\left(\mathrm{TiO}_{2}\right)_{n}(n=1-4)$ clusters showing a structural similarity to the natural anatase phase. ${ }^{6} \mathrm{~W}$. W. Zhang et al. summarized several structural rules of $\left(\mathrm{TiO}_{2}\right)_{n}$ clusters from the perspective of bonding properties and bonding number. ${ }^{7}$ In these previous studies, the influence of the steel matrix on the formation and stability of $\mathrm{TiO}_{x}$ clusters was not taken into consideration. Y. Jiang et al. studied the formation mechanism of $\mathrm{Y}-\mathrm{Ti}-\mathrm{O}$ nanoclusters in bcc Fe lattice,

${ }^{a}$ State Key Laboratory of Advanced Special Steel, Shanghai Key Laboratory of Advanced Ferrometallurgy, School of Materials Science and Engineering, Shanghai University, Shanghai 200072, China.E-mail: lihuigai@shu.edu.cn

${ }^{b}$ Shanghai Institute of Applied Physics, Chinese Academy of Sciences (CAS), Shanghai 201800, China showing that the matrix affected the subsequent precipitations of ( $\mathrm{Y}-\mathrm{Ti}-\mathrm{O})$ nanoclusters by providing limited candidate locations for dissolved solutes. ${ }^{8}$ However, these studies mostly focused on free-standing titanium oxide cluster. The interaction between solute atoms, the structure of titanium oxide clusters in bcc Fe and even their evolution law keep unknown.

In the present work, the interactions between $\mathrm{Ti}, \mathrm{O}$ and $\mathrm{Fe}$ matrix, the structure of $\mathrm{Ti}_{m} \mathrm{O}_{n}$ clusters with $m, n$ ranging from 1 to 4 in bcc-Fe lattice and its possible evolution pathways have been explored using first-principles calculation. The binding energy of different $\mathrm{Ti}_{m} \mathrm{O}_{n}$ clusters was calculated to find the relative stability of the clusters in bcc Fe lattice. The structural evolution pathways were speculated, which was helpful to understand the nucleation of titanium oxides.

\section{Calculation details}

The calculations were carried out using the Vienna $A b$ initio Simulation Package (VASP) ${ }^{9}$ based on the density functional theory (DFT). ${ }^{10}$ To describe the interactions between ions and electrons, standard projector augmented wave pseudopotential $(\mathrm{PAW})^{11}$ were used for $\mathrm{Fe}$, Ti, and $\mathrm{O}$ with $8\left(3 \mathrm{~d}^{6} 4 \mathrm{~s}^{2}\right), 4\left(3 \mathrm{~d}^{4}\right)$, and 6 $\left(2 s^{2} 2 p^{6}\right)$ valence electrons, respectively. Exchange and correlation energy was treated by a generalized gradient approximation with the Perdew-Burke-Ernzerhof functionals (PBE-GGA). ${ }^{12}$ At $0 \mathrm{~K}$, the optimal lattice constant of bcc-Fe was calculated to be $2.83 \AA$ which was consistent with experimental value of $2.85 \AA$ (ref. 13) and other DFT calculations. ${ }^{\mathbf{1 4 1 5}}$ The cutoff energy for the wave expansion was set to be $310 \mathrm{eV}$. A $4 \times 4 \times 4$ supercell of $128( \pm 1, \pm 2, \ldots)$ atoms was used in calculations. All the calculations were spin polarized. In structural optimizations, the shape and size of the supercell and atomic configuration were fully relaxed until the Hellman-Feynman force on all atoms had fallen below $0.01 \mathrm{eV}^{-1}$, while the electronic degrees of 
freedom were converged to at least $10^{-6} \mathrm{eV}$ per atom. The standard conjugate-gradient algorithm implemented in the VASP code was used to relax the ions. A finite-temperature smearing method of first-order $(N=1)$ Methfessel-Paxton was used throughout with the smearing width, $\sigma$, setting to $0.2 \mathrm{eV}$ to keep the difference between the free energy and the total energy negligible (less than $1 \mathrm{meV}$ per atom). Brillouin zone integration was performed with a $3 \times 3 \times 3 k$-point mesh by MonkhorstPack scheme for energy calculation.

To describe the chemical stability of interstitial oxygen atoms and titanium oxide clusters in Fe lattice, two energetic quantities were defined: the equilibrium formation energy and binding energy.

The equilibrium formation energy, $\Delta E_{\mathrm{f}}^{\mathrm{eq}}\left(\mathrm{Fe}_{n} / \mathrm{O}\right)$ was given by the following formula:

$$
\Delta E_{\mathrm{f}}^{\mathrm{eq}}\left(\mathrm{Fe}_{n} / \mathrm{O}\right)=E\left(\mathrm{Fe}_{n} / \mathrm{O}\right)-n E(\mathrm{Fe})-E(\mathrm{O})
$$

where $E\left(\mathrm{Fe}_{n} / \mathrm{O}\right)$ was the total energy of supercell containing $n \mathrm{Fe}$ atoms $(n=128)$ and one oxygen atom, $E(\mathrm{Fe})$ and $E(\mathrm{O})$ were the energy (per atom) of $\mathrm{Fe}$ and $\mathrm{O}$ in their equilibrium pure element reference state. The reference state for $\mathrm{O}$ was the isolated $\mathrm{O}_{2}$ molecule and its energy was determined by conducting a calculation of an $\mathrm{O}_{2}$ molecule in a $10 \AA \times 10 \AA \times 10 \AA$ box. ${ }^{4,16}$ $E(\mathrm{Fe})$ and $E(\mathrm{O})$ were calculated to be $-8.31 \mathrm{eV}(-8.31 \mathrm{eV}$ by C. $\mathrm{M}$. Fang, ${ }^{17}-8.21 \mathrm{eV}$ by C. K. Ande $\left.{ }^{18}\right)$ and $-4.12 \mathrm{eV}(-4.41 \mathrm{eV}$ by $\mathrm{M}$. Souissi ${ }^{19}$ ), respectively.

The binding energy of titanium oxide clusters in Fe lattice, $E_{\mathrm{b}}^{\mathrm{t}}\left(\mathrm{Ti}_{m} \mathrm{O}_{n}\right)$, was defined as follows: ${ }^{20}$

$$
\begin{aligned}
E_{\mathrm{b}}^{\mathrm{t}}\left(\mathrm{Ti}_{m} \mathrm{O}_{n}\right)= & \left\{m E_{\mathrm{t}}\left(\mathrm{Fe}_{N-1} \mathrm{Ti}\right)+n E_{\mathrm{t}}\left(\mathrm{Fe}_{N} \mathrm{O}\right)\right\}-\left\{E_{\mathrm{t}}\left(\mathrm{Fe}_{N-m} \mathrm{Ti}_{m} \mathrm{O}_{n}\right)\right. \\
& \left.+(m+n-1) E_{\mathrm{t}}\left(\mathrm{Fe}_{N}\right)\right\}
\end{aligned}
$$

where $E_{\mathrm{t}}\left(\mathrm{Fe}_{N-1} \mathrm{Ti}\right), E_{\mathrm{t}}\left(\mathrm{Fe}_{N} \mathrm{O}\right)$ and $E_{\mathrm{t}}\left(\mathrm{Fe}_{N-m} \mathrm{Ti}_{m} \mathrm{O}_{n}\right)$ were the total energy of bcc Fe supercell containing a Ti atom, an $\mathrm{O}$ atom and titanium oxide cluster $\mathrm{Ti}_{m} \mathrm{O}_{n}$, respectively. $\mathrm{O}$ atom was considered at interstitial site and $\mathrm{Ti}$ atom at substitutional site. $E_{\mathrm{t}}\left(\mathrm{Fe}_{N}\right)$ was the total energy of perfect bcc Fe supercell. All discussions below obeyed a convention that a positive binding energy indicated a favorable attraction, while a negative one indicated a repulsion.

The binding energy can be decomposed into distortion and electronic binding energy, $\left(E_{\mathrm{b}}^{\mathrm{d}}\left(\mathrm{Ti}_{m} \mathrm{O}_{n}\right), \quad\right.$ and $E_{\mathrm{b}}^{\mathrm{e}}\left(\mathrm{Ti}_{m} \mathrm{O}_{n}\right)$, respectively:

$$
\begin{aligned}
& E_{\mathrm{b}}^{\mathrm{t}}\left(\mathrm{Ti}_{m} \mathrm{O}_{n}\right)=E_{\mathrm{b}}^{\mathrm{d}}\left(\mathrm{Ti}_{m} \mathrm{O}_{n}\right)+E_{\mathrm{b}}^{\mathrm{e}}\left(\mathrm{Ti}_{m} \mathrm{O}_{n}\right) \\
& E_{\mathrm{b}}^{\mathrm{d}}\left(\mathrm{Ti}_{m} \mathrm{O}_{n}\right)=m E_{\mathrm{t}}^{-\mathrm{Ti}}(\mathrm{Fe})+n E_{\mathrm{t}}^{-\mathrm{O}}(\mathrm{Fe})-E_{\mathrm{t}}^{-\left(\mathrm{Ti}_{m} \mathrm{O}_{n}\right)}(\mathrm{Fe}) \\
& -(m+n-1) E_{\mathrm{t}}(\mathrm{Fe})
\end{aligned}
$$

where $E_{\mathrm{t}}^{-\mathrm{Ti}}(\mathrm{Fe}), E_{\mathrm{t}}^{-\mathrm{O}}(\mathrm{Fe})$ and $E_{\mathrm{t}}^{-\left(\mathrm{Ti}_{m} \mathrm{O}_{n}\right)}(\mathrm{Fe})$ were the total energy of the configuration without $\mathrm{Ti}, \mathrm{O}$, and $\mathrm{Ti}_{m} \mathrm{O}_{n}$, respectively. They could be calculated by removing $\mathrm{Ti}, \mathrm{O}$ and $\mathrm{Ti}_{m} \mathrm{O}_{n}$ from fully relaxed Fe supercell containing $\mathrm{Ti}, \mathrm{O}$ and $\mathrm{Ti}_{m} \mathrm{O}_{n}$, respectively. Then the total energy could be calculated without relaxation. The electronic binding energy can be obtained by eqn (3) and (4). The incremental binding energy, $E_{\mathrm{I}}^{\mathrm{X}}\left(\mathrm{Ti}_{m} \mathrm{O}_{n}\right)$, was defined and calculated as:
Table 1 The formation energy $E_{\mathrm{f}}^{\mathrm{eq}}(\mathrm{eV})$, volume change rate $(\% \Delta \varepsilon)$ and relaxation (in percent of $\Delta r_{i} / r_{i}^{0}$ ) for substitutionally and interstitially sited $\mathrm{O}$ atom in bcc Fe. $r_{i}^{0}$ and $r_{i}$ represent the distance between the interstitial impurity atom and its neighboring atoms without relaxation and with relaxation, respectively, $i$ corresponding to the neighbor shell number, i.e. $r_{1}$ is the first nearest neighbor distance

\begin{tabular}{lllll}
\hline Configuration & $E_{\mathrm{f}}^{\text {eq }}$ & $\left(\% \Delta r_{1} / r_{1}^{0}\right)$ & $\left(\% \Delta r_{2} / r_{2}^{0}\right)$ & $(\% \Delta \varepsilon)$ \\
\hline $\mathrm{Fe}_{128} \mathrm{O}$ (oct) & -2.50 & +27.31 & +0.97 & 1.02 \\
$\mathrm{Fe}_{128} \mathrm{O}$ (tet) & -1.77 & +13.35 & +1.23 & 1.46 \\
$\mathrm{Fe}_{127} \mathrm{O}$ (sub) & -0.90 & +2.34 & -2.36 & 0.24 \\
\hline
\end{tabular}

$$
E_{\mathrm{I}}^{\mathrm{X}}\left(\mathrm{Ti}_{m} \mathrm{O}_{n}\right)=E_{\mathrm{b}}^{\mathrm{t}}\left(\mathrm{Ti}_{m} \mathrm{O}_{n}+\mathrm{X}\right)-E_{\mathrm{b}}^{\mathrm{t}}\left(\mathrm{Ti}_{m} \mathrm{O}_{n}\right)
$$

where $\mathrm{X}$ is either Ti or $\mathrm{O}$.

The formation energies of $\mathrm{O}$ atoms in the substitutional site (S-site), octahedral interstitial site (O-site), and tetrahedral interstitial site ( $\mathrm{T}$-site) were calculated. It was found that $\mathrm{O}$ preferred the O-site over the T-site and S-site (see Table 1), which is in agreement with the previously findings. ${ }^{21-23}$

\section{Results and discussion}

Unlike independent atoms, Ti and $\mathrm{O}$ as solute atoms are confined by the Fe matrix. The interaction between solutes were analyzed from the perspective of binding energy and the differential charge density distributions.

The configurations for the pairs in bcc Fe were shown in Fig. 1. The binding energy of $\mathrm{Ti}-\mathrm{Ti}$ and $\mathrm{O}-\mathrm{O}$ pairs and distance between two atoms were calculated and listed in Table 2. The substitutional site inn stands for the first to the fifth nearest neighbors ( $1 \mathrm{nn}$ to $5 \mathrm{nn}$ ) relative to a substitutional solute or an octahedral interstitial in this study. The binding energy between Ti solutes in bcc Fe lattice is negative at the 1nn, 2nn, 3nn, and $4 \mathrm{nn}$ sites and close to 0 at $5 \mathrm{nn}$ site, which means that Ti solute atoms are not liable to aggregate in bcc Fe. The interaction of $\mathrm{O}-\mathrm{O}$ pairs appears strongly attractive in the configuration $\mathrm{BC}$ with a positive binding energy of $0.239 \mathrm{eV},(0.24 \mathrm{eV}$ by C. Barouh et $a .^{22}$ ).

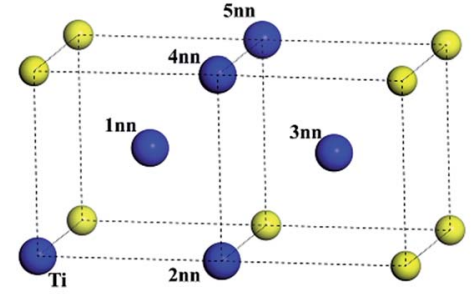

(a)

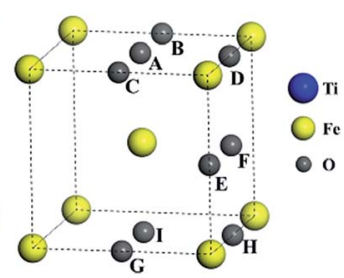

(b)
Fig. 1 Solute-solute configurations in bcc Fe. (a) The first- to fifthnearest-neighbor ( $1 \mathrm{nn}$ to $5 \mathrm{nn}$ ) positions relative to the solute Ti. (b) Possible configurations of two octahedral interstitial $O$ atoms close to each other in bcc lattice. The blue, yellow and gray spheres denote Fe, $\mathrm{Ti}$ and $\mathrm{O}$ atoms, respectively. 
Table 2 The binding energy $(\mathrm{eV})$ of Ti-Ti and O-O pairs and distance ( $\mathrm{A}$ ) between two paired atoms

\begin{tabular}{|c|c|c|c|c|c|}
\hline \multirow[b]{2}{*}{ Different configuration } & \multicolumn{2}{|l|}{$E_{\mathrm{b}}^{\mathrm{t}}(\mathrm{eV})$} & \multirow[b]{2}{*}{ Different configuration } & \multirow{2}{*}{$\frac{E_{\mathrm{b}}^{\mathrm{t}}(\mathrm{eV})}{\mathrm{O}-\mathrm{O}}$} & \multirow[b]{2}{*}{ Distance $(\AA)$} \\
\hline & $\mathrm{Ti}-\mathrm{Ti}$ & & & & \\
\hline $\mathrm{Cfg}(2 n n)$ & -0.251 & 2.912 & Cfg (AI) & -1.457 & 3.441 \\
\hline Cfg (3nn) & -0.183 & 4.031 & $\mathrm{Cfg}(\mathrm{AE})$ & 0.149 & 2.501 \\
\hline $\mathrm{Cfg}(4 \mathrm{nn})$ & -0.012 & 4.726 & $\mathrm{Cfg}(\mathrm{AH})$ & -0.152 & 3.369 \\
\hline & & & Cfg (BE) & -0.050 & 3.502 \\
\hline & & & Cfg (BG) & 0.064 & 3.872 \\
\hline
\end{tabular}

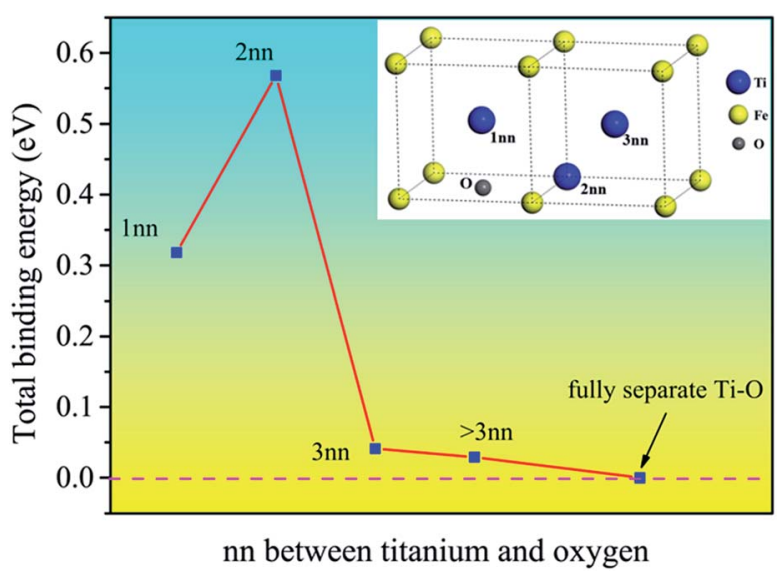

Fig. 2 The binding energy of Ti-O pairs being different nearest neighbor is plotted. The embedded figure shows the corresponding $\mathrm{Ti}-\mathrm{O}$ configuration. It is seen that the present value for $2 \mathrm{nn} \mathrm{Ti-O}$ pair is larger than others, implying that $\mathrm{Ti}$ atom at $2 \mathrm{nn}$ site has high affinity for interstitial $O$ atom.

The binding energies of Ti-O pairs at different nearest neighbor positions were presented in Fig. 2. The results show that the $\mathrm{Ti}$ and $\mathrm{O}$ atoms will attract each other to form Ti-O pairs in bcc Fe. The first and second nearest neighboring Ti-O pairs have quite large positive binding energy, but the value decreases quickly after the second nearest neighbor and is near to zero at the third nearest neighbor, indicating that the interaction is negligible at third nearest neighbor distance. The Ti-O

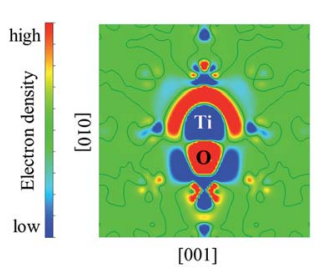

(a)

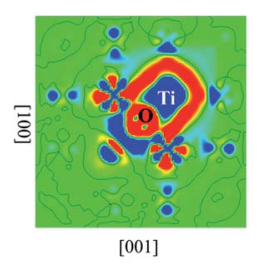

(b)

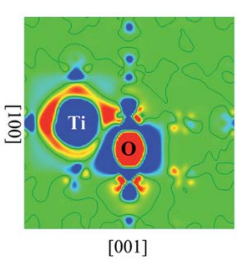

(c)
Fig. 3 The differential charge density distributions for $\mathrm{O}$ with $\mathrm{Ti}$ in substitutional sites of (a) $1 \mathrm{nn}$ in the (100) plane, (b) $2 \mathrm{nn}$ in the (100) plane, (c) $3 n n$ in the (100) plane. Here, the red and blue regions indicate the gain of electrons and the loss of electrons, respectively. The Ti and $\mathrm{O}$ are all labeled. pairs are especially stable when $\mathrm{Ti}$ and $\mathrm{O}$ atoms are at the second nearest neighbor distance. The attractive interaction between $\mathrm{Ti}$ and $\mathrm{O}$ can promote the formation of $\mathrm{Ti}-\mathrm{O}$ clusters in bcc Fe. Nanoscale clusters enriched in $\mathrm{O}$ and Ti had been detected in steel matrix. ${ }^{24,25}$

The differential charge density distributions were calculated to analyze the electronic structures of Ti-O pairs in bcc Fe. It can be seen from Fig. 3 that significant charges transfer from $\mathrm{Ti}$ atoms to $\mathrm{O}$ atoms. The interaction between $\mathrm{Ti}$ and $\mathrm{O}$ presents ionic binding characteristics. There exists certain amount of charges around the Ti-O pair, which means that the pair and the bcc Fe matrix share an amount of charges. Moreover, 2nn Ti-O pair shares the most amount of charges with bcc Fe compared to $1 \mathrm{nn}$ and $3 \mathrm{nn}$ pairs (details see Fig. 3). The result indicated that $\mathrm{Fe}$ matrix contributes to the strong interactions of $2 \mathrm{nn}$.

As a next step, we performed calculations of a number of small $\mathrm{Ti}_{m} \mathrm{O}_{n}$ clusters, ( $m$ and $n$ ranged from 1 to 4 ) to find the most stable structures by comparing total energies among their isomers. Furthermore, we also explored the key factors that influence the stability of structures. The initial nanostructures of titanium oxide clusters were generated according to the following rules: (i) $\mathrm{Ti}$ exists as a substitutional atom, and $\mathrm{O}$ atom prefers to be at an octahedral interstitial site in bcc Fe lattice. (ii) The new structures were constructed by successively adding Ti atom or $\mathrm{O}$ atom to an existed small optimized cluster. (iii) Newly inserted $\mathrm{O}$ atoms and $\mathrm{O}$ atoms in the cluster form the most stable BC configuration. (iv) The Ti-O and Ti-Ti bonds are supposed to form if the distances are smaller than $2.15 \AA$ and $3.00 \AA$, respectively, which are reasonable values compared to the shortest Ti-O distance $(1.95 \AA)$ and Ti-Ti distances $(2.96 \AA)$ in rutile. ${ }^{26}(\mathrm{v})$ Only the first and second nearest neighbor sites to $\mathrm{O}$ atom are replaced by Ti atom to form so-called "compact clusters". Similar rules have also been found in the investigation of stable $\mathrm{Vac}_{n} \mathrm{Al}_{m}$ clusters in $\alpha-\mathrm{Fe},{ }^{27} \mathrm{Vac}^{-\mathrm{As}_{n}}$ clusters in $\mathrm{Ge}^{28}$ and $\mathrm{X}-\mathrm{Pb}_{n}\left(\mathrm{Bi}_{n}\right)$ complex (X $=\mathrm{He}$, Vac, Div) in Fe. ${ }^{29}$

The equilibrium geometries of investigated $\mathrm{Ti}_{m} \mathrm{O}_{n}$ clusters as well as some of their isomers are shown in Fig. 4 . Their energetic and bonding properties are summarized in Table 3. Here, we may reveal several characteristics which can serve as guiding factors to investigate larger clusters by analyzing only a selection of the possible initial clusters. The stability of isomers can be characterized by the total energy. 


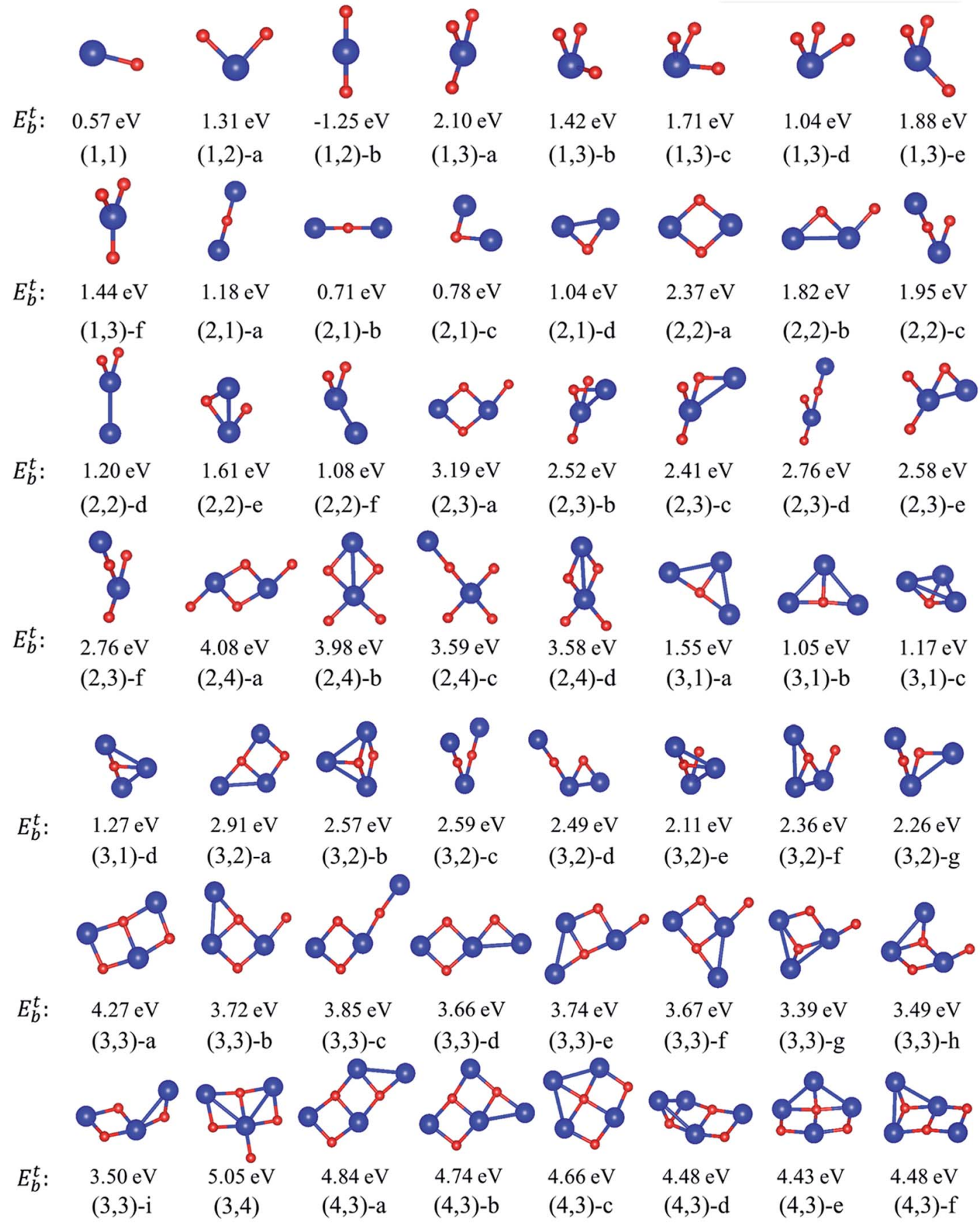

\section{Ti $\bullet O$}

Fig. 4 Schematic representation of the lowest-energy and some of low-lying isomers of $\mathrm{Ti}_{m} \mathrm{O}_{n}$ clusters in bcc lattice ( $m, n$ ranging from 1 to 4 ). The $E_{b}^{t}$ represents total binding energy. It should be note that the structure is labeled by the symbol of $(m, n)$-a is the most stable configuration among its isomers, e.g., $(m, n)-b$, or $(m, n)-c$, etc., where $m$ and $n$ represent the number of Ti atoms (blue spheres) and $O$ atoms (red spheres), respectively. Fe atoms are not drawn here.

It has been found that the formation of $\mathrm{Ti}-\mathrm{O}$ bonds helps to promote the stability of clusters on account of strong attractive interactions between $\mathrm{Ti}$ and $\mathrm{O}$. As can be seen in Table 3, the most stable structures $(m, n)$-a are those with the largest amount of Ti-O bonds among their isomers. However, it should be noted that the first-nearest-neighbor $\mathrm{Ti}-\mathrm{O}$ bond induces the total energy to increase slightly, i.e. the total energy of the structures $(3,1)$-a, $(3,1)$-b and $(3,1)$-d increase sequentially as the number of first-nearest-neighbor Ti-O bonds increase. Similarly, the structure $(3,2)$-d is more stable than $(3,2)$-g due to lack 
Table 3 The total energy relative to the corresponding the lowest energy isomers (take to be zero) $E$ (eV), the number of Ti-Ti, $\mathrm{Ti}-\mathrm{O}$ and $\mathrm{Ti}-\mathrm{O}$ (which is first nearest neighbor, $1 \mathrm{nn})$ bonds $\left(N_{(\mathrm{Ti}-\mathrm{Ti})}, N_{(\mathrm{Ti}-\mathrm{O})}\right.$ and $N_{(\mathrm{Ti}-\mathrm{O})}-1 \mathrm{nn}$ respectively) of $\mathrm{Ti}_{m} \mathrm{O}_{n}$ clusters for $m$ and $n$ up to 4

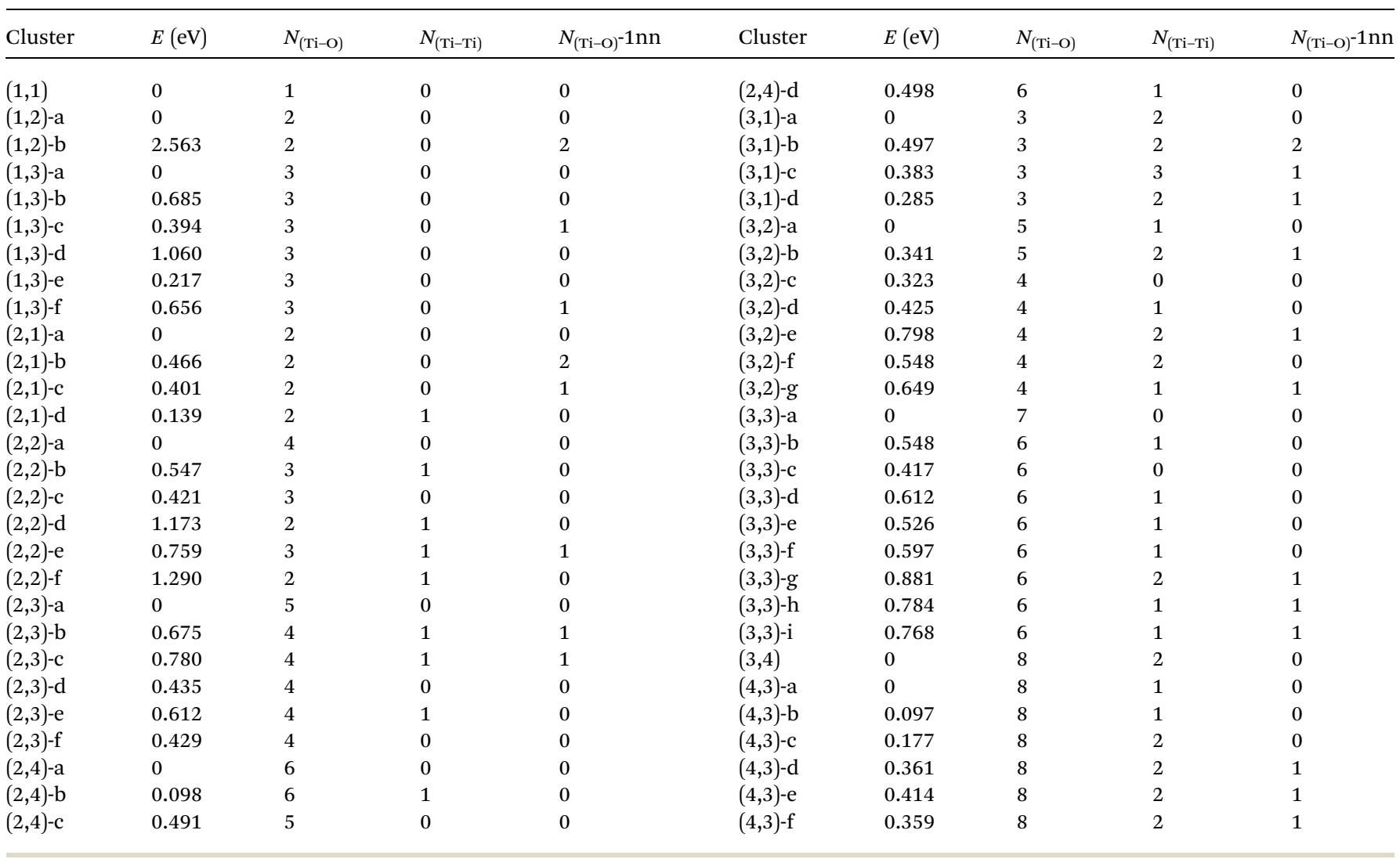

of one first-nearest-neighbor Ti-O bond. The repulsive interaction between Ti-Ti influences the total energy, resulting in less stability of the structures. For example, the structure $(2,4)$-a, which is similar to the most stable structure predicted in previous calculations, ${ }^{6,30}$ is more stable than structure $(2,4)$ $\mathrm{b}$ due to the smaller distance between Ti atoms. The least stable structure $(3,3)$-g in $(3,3)$ isomers contains two Ti-Ti bonds. For $(4,3)$ type clusters, they all have eight Ti-O bonds. Consequently, the structure with the least Ti-Ti and first-nearestneighbor Ti-O bonds has the lowest energy. Besides, the most stable structures such as (2,2)-a and (3,3)-a, are all ring-shaped, and are more energetically favorable to form than quasi-linear structures.

Based on the stable structures for various clusters analyzed above, next we turn to discuss the possible growth pathway of $\mathrm{Ti}_{m} \mathrm{O}_{n}$ clusters in the initial stage of nucleation.

The binding energy for the stable clusters of different sizes was calculated. The results are plotted in Fig. 5 . It can be seen that the binding energies of the clusters are positive, which means that the $\mathrm{Ti}_{m} \mathrm{O}_{n}$ clusters are all energetically favorable to form. This indicates that small clusters may grow up by absorbing $\mathrm{Ti}$ or $\mathrm{O}$ atoms into a larger cluster, which is consistent with the previous thermodynamic calculation. ${ }^{31}$

To explore the origin of the binding energy, we decomposed the binding energy into the distortion and electronic binding energy. The decomposed energy together with the binding energy for the $\mathrm{Ti}_{m} \mathrm{O}_{n}$ clusters are plotted in Fig. 6. It can be seen that $E_{\mathrm{b}}^{\mathrm{d}}\left(\mathrm{Ti}_{m} \mathrm{O}_{n}\right)$ increases with the size of the $\mathrm{Ti}_{m} \mathrm{O}_{n}$ cluster and becomes positive when $m=n=2$ (the rhombus-shaped structure forms). The result indicates that the strain in matrix caused by the cluster can be relaxed and the cluster is liable to grow up when the $\mathrm{Ti}_{2} \mathrm{O}_{2}$ rhombus-shaped structure forms. The electronic binding energy, $E_{\mathrm{b}}^{\mathrm{e}}\left(\mathrm{Ti}_{m} \mathrm{O}_{n}\right)$, is also positive and increases with the size of the clusters. $E_{\mathrm{b}}^{\mathrm{e}}\left(\mathrm{Ti}_{m} \mathrm{O}_{n}\right)$ is larger than the distortion binding energy, $E_{\mathrm{b}}^{\mathrm{d}}\left(\mathrm{Ti}_{m} \mathrm{O}_{n}\right)$, and mainly contributes to the binding energy.

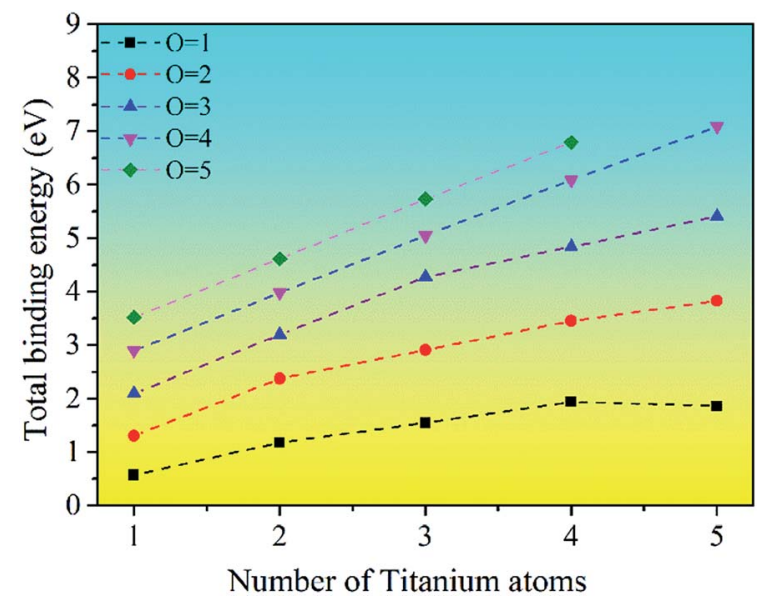

Fig. 5 The binding energy (in eV) of titanium oxide clusters $\left(\mathrm{Ti}_{m} \mathrm{O}_{n}\right)$. 


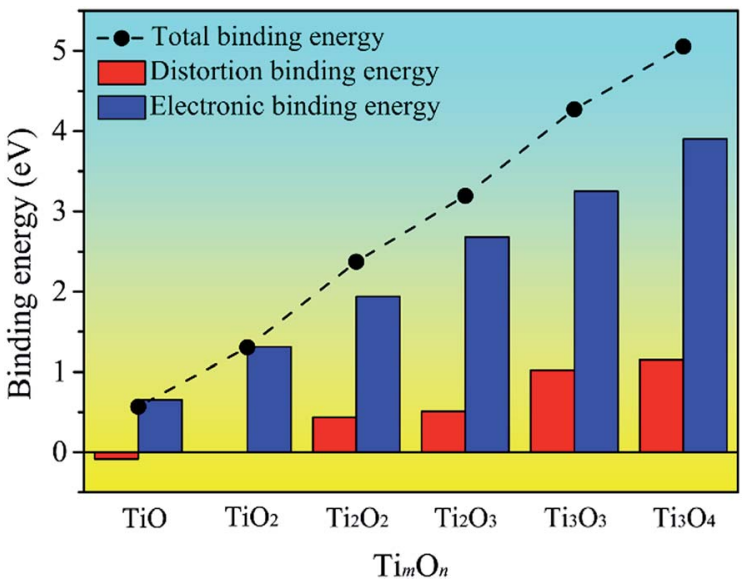

Fig. 6 Decomposition of the binding energy (in eV) into distortion binding energy and electronic binding energy for the given most stable titanium oxide clusters $\left(\mathrm{Ti}_{m} \mathrm{O}_{n}\right)$. Binding energy are shown as dashed line.

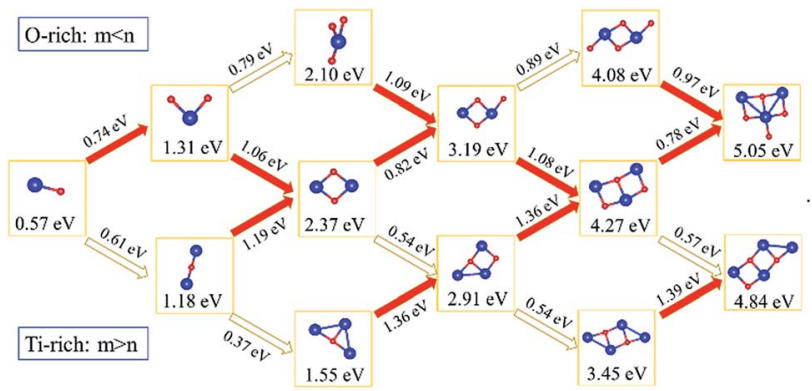

Fig. 7 Schematic representation of structural evolution for small $\mathrm{Ti}_{m} \mathrm{O}_{n}$ clusters. The values (in eV) given under the cluster are the corresponding binding energy. The incremental binding energy is attached on the arrows which predicted the next probable structure. Noted that the clusters arrowed by red arrows (with larger incremental binding energy than blank arrows) have an even greater possibility to appear ( $m, n$ denote the number of titanium and oxygen, respectively).

Subsequently, we explored the structural evolution of small $\mathrm{Ti}_{m} \mathrm{O}_{n}$ clusters (see Fig. 7). Only the relatively stable structures of each size of clusters are discussed here. The incremental binding energy, $E_{\mathrm{I}}^{\mathbf{x}}\left(\mathrm{Ti}_{m} \mathrm{O}_{n}\right)$, represents the binding energy between an $\mathrm{X}$ (Ti or $\mathrm{O}$ ) atom and a $\mathrm{Ti}_{m} \mathrm{O}_{n}$ cluster. TiO cluster prefers to form $\mathrm{TiO}_{2}$ for the reason that $E_{\mathrm{I}}^{\mathrm{O}}(\mathrm{TiO})$ is larger than $E_{\mathrm{I}}^{\mathrm{Ti}}(\mathrm{TiO})$, but it is also undeniable that the structure $\mathrm{Ti}_{2} \mathrm{O}$ may exist. Both $\mathrm{TiO}_{2}$ and $\mathrm{Ti}_{2} \mathrm{O}$ preferentially form the rhombusshaped structure, $\mathrm{Ti}_{2} \mathrm{O}_{2}$, by trapping one $\mathrm{Ti}$ atom and one $\mathrm{O}$ atom, respectively. The structures formed by O-rich cluster $\mathrm{TiO}_{3}$ and Ti-rich cluster $\mathrm{Ti}_{3} \mathrm{O}$ after trapping a $\mathrm{Ti}$ and $\mathrm{O}$ atom, respectively, also contain a rhombus-shaped structure $\mathrm{Ti}_{2} \mathrm{O}_{2}$. However, $E_{\mathrm{I}}^{\mathrm{O}}\left(\mathrm{Ti}_{2} \mathrm{O}_{2}\right)$ is larger than $E_{\mathrm{I}}^{\mathrm{Ti}}\left(\mathrm{Ti}_{2} \mathrm{O}_{2}\right)$, indicating that the cluster $\mathrm{Ti}_{2} \mathrm{O}_{2}$ preferentially traps one $\mathrm{O}$ atom, forming a terminal Ti-O bond. The structure of $\mathrm{Ti}_{2} \mathrm{O}_{3}$ is similar to the structure of $\mathrm{Ti}_{3} \mathrm{O}_{2}$, both containing a quadrangular configuration, while these two clusters preferentially form the same cluster $\mathrm{Ti}_{3} \mathrm{O}_{3}$ containing two quadrangular units which in conjunction with each other by sharing one Ti-O bond. Besides, the cluster $\mathrm{Ti}_{2} \mathrm{O}_{4}$ and the cluster $\mathrm{Ti}_{4} \mathrm{O}_{2}$ prefer to bond one $\mathrm{Ti}$ atom and $\mathrm{O}$ atom, respectively, tending to make the stoichiometric ratio of the cluster approach to a value of $1: 1$. This is in excellent agreement with our experimental finding that the Ti to O ratio approaches to $1: 1$ for clusters detected by Atom Probe Tomography (APT). It is noted that the matrix Fe atoms surrounding the constructed $\mathrm{Ti}_{m} \mathrm{O}_{n}$ clusters present short-range ordered stacking, having effects on the structures of clusters. Take the process from $\mathrm{Ti}_{2} \mathrm{O}_{2}$ to $\mathrm{Ti}_{3} \mathrm{O}_{3}$ for example (see Fig. 8). Before formation of clusters, $\mathrm{Ti}$ and $\mathrm{O}$ atoms are considered distributing stochastically in forms of free atoms or Ti-O pairs in the $\mathrm{Fe}$ melt. One $\mathrm{O}$ atom firstly migrates toward an as-formed $\mathrm{Ti}_{2} \mathrm{O}_{2}$ and then $\mathrm{Ti}_{2} \mathrm{O}_{2}$ develops into $\mathrm{Ti}_{2} \mathrm{O}_{3}$. But the $\mathrm{O}$ atom is restricted by $\mathrm{Fe}$ matrix lattices, which are supposed to offer limited O-sites for $\mathrm{O}$ to select when it bonds with Ti atom. The free $\mathrm{Ti}$ atom afterwards occupies the lattice point to bond with two $\mathrm{O}$ atoms and then rhombus-shaped structure forms. So, the calculated structures of $\mathrm{Ti}_{m} \mathrm{O}_{n}$ clusters are slightly different from other calculations in bonding characteristics. ${ }^{32-34}$

Based on the study of these small clusters, we can conclude that Ti-rich clusters, i.e. $\mathrm{Ti}_{2} \mathrm{O}, \mathrm{Ti}_{3} \mathrm{O}, \mathrm{Ti}_{3} \mathrm{O}_{2}$ and $\mathrm{Ti}_{4} \mathrm{O}_{2}$, are especially apt to trap $\mathrm{O}$ atom, while $\mathrm{O}$-rich clusters, i.e. $\mathrm{TiO}_{2}, \mathrm{TiO}_{3}$, $\mathrm{Ti}_{2} \mathrm{O}_{3}$ and $\mathrm{Ti}_{2} \mathrm{O}_{4}$, are apt to trap Ti atom. This indicates that the clusters whose ratio of Ti to $\mathrm{O}$ atoms is close to $1: 1$ are easily to be formed, and then these clusters evolve towards structures containing more $\mathrm{O}$ atoms. This tendency is consistent with the experimental results that some common titanium oxides inclusions, i.e. $\mathrm{TiO}_{2}, \mathrm{Ti}_{2} \mathrm{O}_{3}$, and $\mathrm{Ti}_{3} \mathrm{O}_{5}$ etc., contain more $\mathrm{O}$ atoms than $\mathrm{Ti}$ atoms. ${ }^{34,35}$ Additionally, the structure (2,2)-a was regarded as a special cluster, which may be considered as primitive or potential nuclei of stable and larger titanium oxides. For instance, we found this rhombus-shaped structure in several common natural phases, i.e. $\mathrm{Ti}_{2} \mathrm{O}_{3}$ and $\mathrm{TiO}_{2}$ (including three bulk structures, anatase, brookite and rutile). For the structure of $\mathrm{Ti}_{2} \mathrm{O}_{3}$, one Ti atom is surrounded by six $\mathrm{O}$ atoms, forming three rhombus-shaped structures sharing one

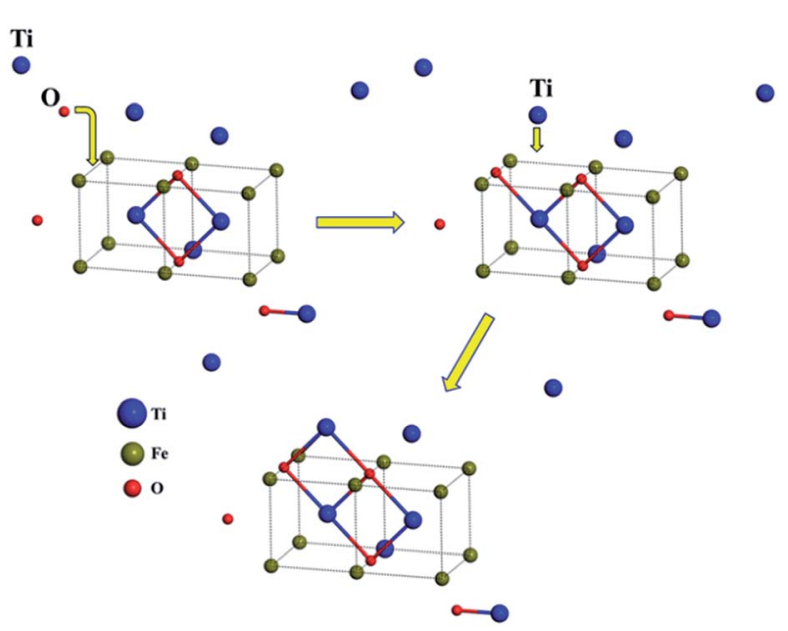

Fig. 8 Schematic representation of structural evolution for $\mathrm{Ti}_{2} \mathrm{O}_{2}$ to $\mathrm{Ti}_{3} \mathrm{O}_{3}$ (blue, green and red spheres denote $\mathrm{Ti}, \mathrm{Fe}$ and $\mathrm{O}$ atoms respectively). 


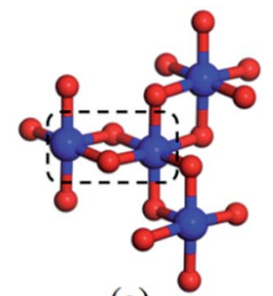

(a)

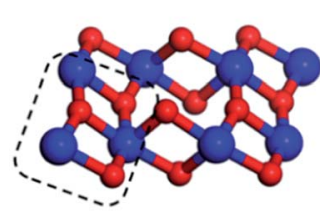

(c)

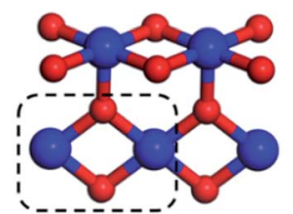

(b)

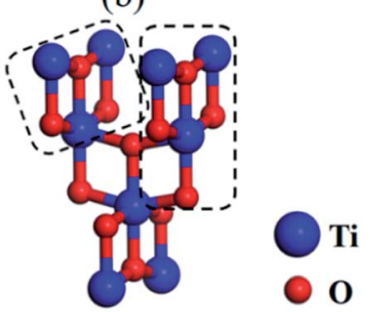

(d)
Fig. 9 Schematic view of several common bulk structures of titanium oxides for (a) $\mathrm{Ti}_{2} \mathrm{O}_{3}$, (b) $\mathrm{TiO}_{2}$ (rutile), (c) $\mathrm{TiO}_{2}$ (brookite) and (d) $\mathrm{TiO}_{2}$ (anatase). Noted that the rhombus-shaped structures in the dashed boxes are similar to a selection of clusters shown in Fig. 8. They comprise these bulk structures by complicated evolving (blue and red spheres denote $\mathrm{Ti}$ and $\mathrm{O}$ atom, respectively).

$\mathrm{Ti}$ atom, (see Fig. 9a). In rutile $\mathrm{TiO}_{2}$, one $\mathrm{Ti}$ atom bridged two adjacent rhombus-shaped structures lying on one plane (see Fig. 9b). For brookite and anatase, the small units selected by dashed boxes were similar to the clusters of $(3,3)$-a and $(3,4)$ (compare Fig. 9c, d and 4). The rhombus-shaped structure can be considered to be the basic unit of the stable $\mathrm{Ti}_{m} \mathrm{O}_{n}$ structure in Fe matrix. The fact that these clusters studied theoretically may also be useful for us to understand the structural evolution further.

\section{Conclusions}

The Ti and $\mathrm{O}$ are known to mainly exist as solute atoms in the medium-range-order bcc Fe matrix and can be easily to aggregate and form clusters due to the strongly attractive interaction between them. The growing pathway and the origin of $\mathrm{Ti}_{m} \mathrm{O}_{n}$ clusters are explored from the changes of binding energy. The detailed conclusions are as follows:

(i) The interaction between a substitutional $\mathrm{Ti}$ and an interstitial $\mathrm{O}$ is strongly attractive at the second neighbor distance and presents ionic binding characteristics. A sizeable amount of charges are shared by $\mathrm{Ti}-\mathrm{O}$ pairs and Fe matrix when $\mathrm{Ti}$ and $\mathrm{O}$ at second neighbor distance.

(ii) The Ti-O, Ti-Ti bonds are the main factors affecting the stability of clusters, which are more stable with increasing number of $2 \mathrm{nn} \mathrm{Ti}-\mathrm{O}$ bonds or decreasing number of $\mathrm{Ti}-\mathrm{Ti}$ bonds.

(iii) The O-rich clusters are energetically favorable to trap $\mathrm{Ti}$ atom and the Ti-rich clusters tend to trap $\mathrm{O}$, to keep the stoichiometric ratio being close to $1: 1$, and then the cluster tends to absorb $\mathrm{O}$ atom.

(iv) The rhombus-shaped structure $(2,2)$-a is suggested to be the smallest unit of the stable $\mathrm{Ti}_{m} \mathrm{O}_{n}$ structure in Fe matrix.

\section{Conflicts of interest}

We declare that we do not have any commercial or associative interest that represents a conflict of interest in connection with this work.

\section{Acknowledgements}

This work is supported by National Natural Science Foundation of China (No. U1460103), High Performance Computing Center, Shanghai University. The authors would like to express gratitude to Dr Y. P. Xie from Shanghai University for the valuable assistance during the present study.

\section{Notes and references}

1 J. S. Byun, J. H. Shim, Y. W. Cho and D. N. Lee, Acta Mater., 2003, 51, 1593-1606.

2 Y. Li, X. L. Wan, L. Cheng and K. M. Wu, Mater. Sci. Technol., 2016, 32, 88-93.

3 W. Z. Mu, P. G. Jönsson and K. Nakajima, J. Mater. Sci., 2016, 51, 2168-2180.

4 Y. Guo, J. F. Li, X. Niu, A. Markovits and R. Zhang, Phys. Chem. Chem. Phys., 2016, 18, 10594-10599.

5 Z. W. Qu and G. J. Kroes, J. Phys. Chem. B, 2006, 110, 89989007.

6 S. Li and D. A. Dixon, J. Phys. Chem. A, 2008, 112, 6646-6666.

7 W. W. Zhang, Y. Han, S. Yao and H. Sun, Mater. Chem. Phys., 2011, 130, 196-202.

8 Y. Jiang, J. R. Smith and G. R. Odette, Phys. Rev. B, 2009, 79, 064103.

9 G. Kresse and J. Hafner, Phys. Rev. B, 1994, 49, 14251.

10 P. Hohenberg and W. Kohn, Phys. Rev., 1964, 136, 864.

11 G. Kresse and D. Joubert, Phys. Rev. B, 1999, 59, 1758.

12 J. P. Perdew, K. Burke and M. Ernzerhof, Phys. Rev. Lett., 1996, 77, 3865.

13 Y. Wang, S. Curtarolo, C. Jiang, R. Arroyave, T. Wang, G. Ceder, L. Q. Chen and Z. K. Liu, Calphad, 2004, 28, 79-90.

14 P. K. Nandi, et al., J. Phys.: Condens. Matter, 2010, 22, 345501.

15 Y. P. Xie and S. J. Zhao, Comput. Mater. Sci., 2012, 63, 329335.

16 W. A. Counts, C. Wolverton and R. Gibala, Acta Mater., 2010, 58, 4730-4741.

17 C. M. Fang, M. Van Huis, M. Sluiter and H. Zandbergen, Acta Mater., 2010, 58, 2968-2977.

18 C. K. Ande and M. H. Sluiter, Acta Mater., 2010, 58, 62766281.

19 M. Souissi and H. Numakura, Comput. Mater. Sci., 2016, 124, 249-258.

20 C. Domain, C. S. Becquart and J. Foct, Phys. Rev. B, 2004, 69, 144112.

21 C. L. Fu, M. Krčmar, G. S. Painter and X. Q. Chen, Phys. Rev. Lett., 2007, 99, 225502.

22 C. Barouh, T. Schuler, C. C. Fu and M. Nastar, Phys. Rev. B, 2014, 90, 054112. 
23 S. L. Shang, H. Z. Fang, J. Wang, C. P. Guo, Y. Wang, P. D. Jablonski, Y. Du and Z. K. Liu, Corros. Sci., 2014, 83, 94-102.

24 M. K. Miller, et al., J. Mater. Sci. Eng. A, 2003, 353, 140-145.

25 M. K. Miller, D. T. Hoelzer, E. A. Kenik and K. F. Russell, Intermetallics, 2005, 13, 387-392.

26 J. Muscat, V. Swamy and N. M. Harrison, Phys. Rev. B, 2002, 65, 224112.

27 H. Amara, C. C. Fu, F. Soisson and P. Maugis, Phys. Rev. B, 2010, 81, 174101.

28 A. Chroneos, R. W. Grimes, B. P. Uberuaga, S. Brotzmann and H. Bracht, Appl. Phys. Lett., 2007, 91, 192106.
29 Y. Zhang, et al., Phys. Chem. Chem. Phys., 2015, 17, 1229212298.

30 T. Albaret, F. Finocchi and C. Noguera, J. Chem. Phys., 2000, 113, 2238-2249.

31 C. X. Chen, H. Xue, H. Peng, L. Yan, L. Zhi and S. Wang, J. Nanomater., 2014, 2014, 1.

32 K. Jeong, C. Chang, E. Sedlmayr and D. Sülzle, J. Phys. B: At., Mol. Opt. Phys., 2000, 33, 3417.

33 S. Hamad, et al., J. Phys. Chem. B, 2005, 109, 15741-15748.

34 J. J. Pak, et al., ISIJ Int., 2007, 47, 16-24.

$35 \mathrm{~J}$. Y. Chen, et al., Advances in the Science and Engineering of Casting Solidification, 2015, vol. 2015, p. 147. 